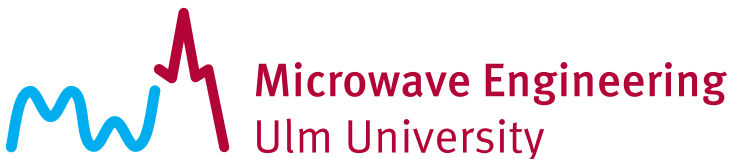

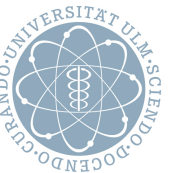

\section{Compensation of Motion-Induced Phase Errors in TDM MIMO Radars}

\author{
Jonathan Bechter, Fabian Roos, and Christian Waldschmidt
}

(C) 2017 IEEE. Personal use of this material is permitted. Permission from IEEE must be obtained for all other uses, in any current or future media, including reprinting/republishing this material for advertising or promotional purposes, creating new collective works, for resale or redistribution to servers or lists, or reuse of any copyrighted component of this work in other works. 


\title{
Compensation of Motion-Induced Phase Errors in TDM MIMO Radars
}

\author{
Jonathan Bechter, Fabian Roos, and Christian Waldschmidt
}

\begin{abstract}
For high-resolution direction of arrival estimation, a wide aperture is necessary. This can be achieved with multipleinput multiple-output (MIMO) radars, which offer a wide virtual aperture. However, the requirement of orthogonal transmit signals has to be satisfied for their operation. Although time division multiplexing (TDM) of the transmit elements is an orthogonality realization with low hardware effort, phase errors occur in nonstationary scenarios. This work briefly discusses the problem of the motion-induced phase errors and describes processing steps to mitigate them without additional effort. The proposed technique is demonstrated with simulation and measurement data.
\end{abstract}

\section{INTRODUCTION}

The current development in automotive radar is going towards multiple-input multiple-output (MIMO) systems, consisting of $M$ transmitters and $N$ receivers. They offer a large number of virtual antenna elements and high angular resolution, and this comes with a reduced effort of hardware and aperture size compared to conventional systems. However, the realization requires the transmission of orthogonal signals. In linear frequency modulated chirp sequence radars, this is typically done with time, frequency, or code division multiplexing. This paper focuses on the time division multiplexing (TDM) scheme, which is used very often, e.g. [1], [2], [3], [4].

A chirp sequence radar transmits a series of linear frequency ramps. The baseband time samples of each chirp are stored in a matrix, and range and velocity are extracted with a twodimensional Fourier transform. This leads to a range-Doppler spectrum for each single channel. When a TDM MIMO scheme is applied, the active transmit element is changed after each transmission of a single chirp. An example with two transmitters $(M=2)$ is shown in Fig. 1. The geometric location of each transmitter and each receiver forms a virtual array which has a maximum number of $M \cdot N$ virtual elements. A range-Doppler spectrum is found for each element of the virtual array.

The phase difference between the antenna elements is used for direction of arrival estimation. In a non-MIMO linear array, the phase difference of a signal on two receive channels is

$$
\Delta \varphi=k d \sin \vartheta,
$$

where $\vartheta$ is the incident angle of the signal, $k$ the wave number, and $d$ the distance between the elements. In a TDM MIMO system as shown in Fig. 1, an additional phase difference has

J. Bechter, F. Roos, and C. Waldschmidt are with the Institute of Microwave Engineering, Ulm University, 89081 Ulm, Germany (e-mail: firstname.lastname@uni-ulm.de).

Manuscript received May 8, 2017; revised August 10, 2017; accepted September 6, 2017. Date of publication?

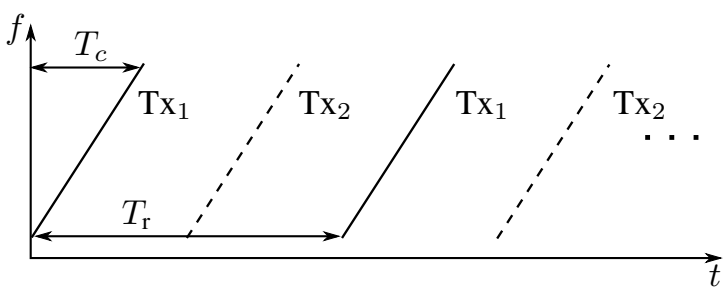

Fig. 1. In the exemplary TDM MIMO scheme, transmitters $\mathrm{Tx}_{1}$ and $\mathrm{Tx}_{2}$ operate in an alternating way. $T_{r}$ limits the unambiguous Doppler frequency [5].

to be considered because of the switching time $T_{\mathrm{r}} / 2$ between the transmitters $\mathrm{Tx}_{1}$ and $\mathrm{Tx}_{2}$. This alters (1) to

$$
\Delta \tilde{\varphi}=k d \sin \vartheta+2 \pi f_{D} T_{\mathrm{r}} / 2 .
$$

Target motion results in a Doppler frequency $f_{D}$ that introduces an additional phase term. For objects with a high $f_{D}$, $\Delta \tilde{\varphi}$ is strongly influenced by this error. In general, for $M$ transmitters the phase relation at the $m$-th transmitter $\operatorname{Tx}_{m}$ is

$$
\Delta \tilde{\varphi}_{\mathrm{Tx}_{m}}=k d \sin \vartheta+2 \pi f_{D} T_{\mathrm{r}} \frac{m-1}{M} .
$$

For the compensation of the motion-induced phase error, [6] proposes to create overlapping elements in the virtual aperture. These elements are used to estimate and correct the error; however, this comes at the cost of $(M-1)$ unique virtual elements and therefore reduces the maximum aperture size or maximum number of channels. Optimizing the switching scheme of the transmitters to reduce the phase error is discussed in [7]. [8] introduces an estimation and interpolation of the motioninduced error. Another approach is the error reduction through an interlaced transmission of frequency ramps [9].

In this paper a straight-forward way is introduced and demonstrated for handling the motion-induced phase error with basic signal processing techniques for a chirp sequence radar. This approach does not require any additional hardware effort and has only small processing requirements.

\section{PHASE ERROR COMPENSATION}

In this section, the origin of the motion-induced phase error is described, and the discrete Fourier transform (DFT) for the extraction of velocity is adjusted to mitigate the phase error.

The baseband time signal of a single frequency chirp $l=0,1,2, \ldots$ is modeled similarly to [5] as

$$
s_{\mathrm{Tx}_{m}}(t, l)=\mathrm{e}^{j\left(2 \pi\left[\frac{2 f_{c} R}{c_{0}}+\left(f_{R}+f_{D}\right) t+l f_{D} T_{\mathrm{r}}\right]+\Delta \tilde{\varphi}_{\mathrm{Tx}_{m}}\right)} .
$$

$c_{0}$ is the speed of light and $f_{c}$ the center frequency of the chirps and $R$ the target distance. The range-dependent frequency is $f_{R}=\frac{2 B R}{c_{0} T_{c}}$. For chirps with a short time duration $T_{c}$ and a high 


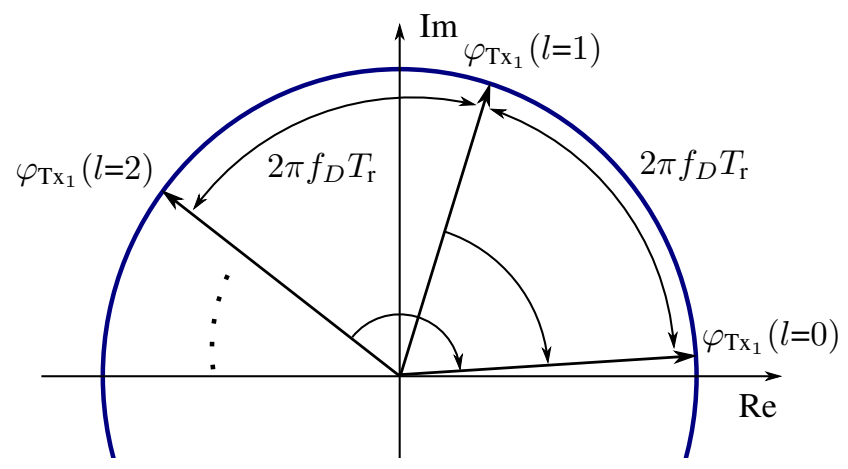

Fig. 2. In a system with a single transmitter, the phase difference of a single target between two successive chirps is $2 \pi f_{D} T_{\mathrm{r}}$. For extraction of the Doppler frequency, the Fourier transform rotates all vectors to the phase $\varphi_{\operatorname{Tx}_{1}}(l=0)$, indicated with arrows. The constructive superposition of the vectors leads to a peak in the spectrum at $f_{D}$.

bandwidth $B$, it is typically assumed that $f_{R} \gg f_{D}$. A Fourier transform $\mathcal{F}\left(s_{\mathrm{Tx}_{m}}(t, l)\right)$ leads to the range spectrum of chirp $l$ :

$$
\begin{aligned}
S_{\mathrm{Tx}_{m}}(f, l) & \propto \operatorname{sinc}\left(\pi T_{c}\left(f-f_{R}\right)\right) e^{j 2 \pi\left[\frac{2 f_{c} R}{c_{0}}+l f_{D} T_{\mathrm{r}}\right]+j \Delta \tilde{\varphi}_{\mathrm{Tx}_{m}}} \\
& =\operatorname{sinc}\left(\pi T_{c}\left(f-f_{R}\right)\right) e^{j \varphi_{\mathrm{T}_{m}}(l)}
\end{aligned}
$$

Fig. 2 shows the complex vector $S_{\mathrm{Tx}_{1}}\left(f_{R}, l\right)$ for a single target at multiple frequency chirps for the case of a single transmitter $\mathrm{Tx}_{1}$. In this non-MIMO setup, a second Fourier transform is calculated across all chirps to extract the velocity. For the frequency $f_{D}$, the Fourier transform rotates all vectors to the phase $\varphi_{\mathrm{Tx}_{1}}(l=0)$, what leads to a maximum:

$$
S_{\text {Doppler, } \mathrm{Tx}_{1}}\left(f_{D}\right)=\sum_{l} \mathrm{e}^{j \varphi_{\mathrm{Tx}_{1}}(l)} \cdot \mathrm{e}^{-j 2 \pi f_{D} T_{\mathrm{r}} l} .
$$

In the TDM MIMO case a second transmitter $\mathrm{Tx}_{2}$ is present. As depicted in Fig. 1 the active transmitter is switched after each successive chirp. Without loss of generality, it is assumed that $\vartheta=0$. Fig. 3 shows how the DFT transforms all vectors corresponding to $\mathrm{Tx}_{2}$ to the phase $\varphi_{\mathrm{Tx}_{2}}(l=0)$. All vectors corresponding to $\mathrm{Tx}_{1}$ still behave as shown in Fig. 2. $\Delta \tilde{\varphi}_{\mathrm{Tx}_{2}}$ introduces the motion-induced systematic error $\varphi_{\text {err }}=2 \pi f_{D} T_{\mathrm{r}} / 2$. In order to mitigate this error, the Doppler processing is changed. The chirps according to $\mathrm{Tx}_{1}$ are still processed with the normal DFT (6). For the chirps transmitted by $\mathrm{Tx}_{2}$, the DFT is adjusted to

$$
S_{\text {Doppler, } \operatorname{Tx}_{2}}\left(f_{D}\right)=\sum_{l} \mathrm{e}^{j \varphi_{\mathrm{TX}_{2}}(l)} \cdot \mathrm{e}^{-j 2 \pi f_{D} T_{\mathrm{r}}(l+1 / 2)} .
$$

With this slightly adapted DFT, the phases of the chirps of $\mathrm{Tx}_{2}$ are also transformed to the phase $\varphi_{\mathrm{Tx}_{1}}(l=0)$. For $\vartheta \neq 0$, a constant phase according to (1) is added to $\varphi_{\mathrm{Tx}_{2}}(l)$. As this phase shift is independent of $l$, it can be extracted from the sum in (7). Thus, the proposed processing is valid for any $\vartheta$. A related way of processing for the reduction of Doppler ambiguities is presented in [10].

Extending the scheme for a number of $M$ transmitters, the Doppler DFT for transmitter $\mathrm{Tx}_{m}$ is calculated by

$$
S_{\text {Doppler, } \operatorname{Tx}_{m}}\left(f_{D}\right)=\sum_{l} \mathrm{e}^{j \varphi_{\mathrm{T}_{m}}(l)} \cdot \mathrm{e}^{-j 2 \pi f_{D} T_{\mathrm{r}}\left(l+\frac{m-1}{M}\right)} .
$$

Because of the linearity of the DFT, (8) also holds in multi-

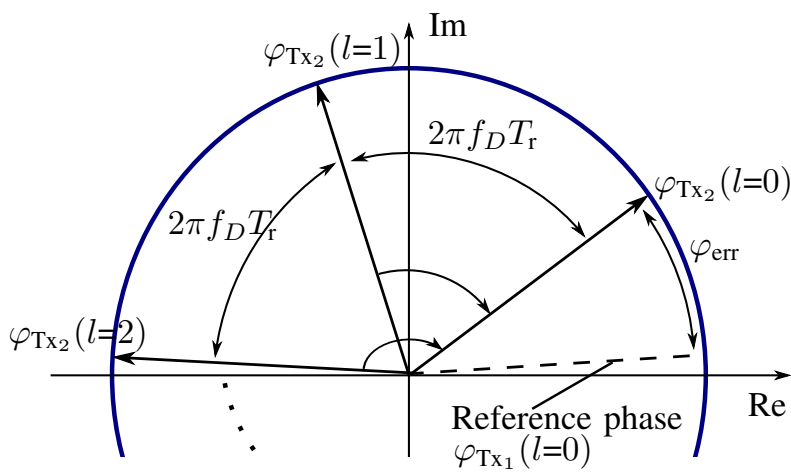

Fig. 3. In a TDM MIMO radar, the chirps of each transmitter are processed independently. While the DFT for $\mathrm{Tx}_{1}$ transforms all phases to $\varphi_{\mathrm{Tx}_{1}}(l=0)$, the phases of $\operatorname{Tx}_{2}$ are transformed to $\varphi_{\mathrm{Tx}_{2}}(l=0)$. This leads to the systematic phase error $\varphi_{\mathrm{err}}=2 \pi f_{D} T_{\mathrm{r}} / 2$.

TABLE I

Modulation Parameters for Simulation and Measurement.

\begin{tabular}{l|r|r} 
& Simulation & Measurement \\
\hline Bandwidth $B$ & $1 \mathrm{GHz}$ & $594 \mathrm{MHz}$ \\
Ramp duration $T_{c}$ & $20 \mu \mathrm{s}$ & $20.480 \mu \mathrm{s}$ \\
Ramp repetition rate $T_{\mathrm{r}}$ & $25 \mu \mathrm{s}$ & $27.015 \mu \mathrm{s}$ \\
Center frequency $f_{c}$ & $77 \mathrm{GHz}$ & $76.41 \mathrm{GHz}$ \\
Number $L$ of frequency chirps & 128 & 128
\end{tabular}

target scenarios. Note that this way of processing is equivalent to an interlaced zero-padding in Doppler dimension prior to conventional DFT processing. This means the input of a conventional DFT is the vector

$$
[S(f, l=0), 0, S(f, l=1), 0, \ldots]
$$

for $\mathrm{Tx}_{1}$, and for $\mathrm{Tx}_{2}$ it is

$$
[0, S(f, l=0), 0, S(f, l=1), \ldots] .
$$

\section{Simulation AND MEASUREMENT}

The simulations compare the direction of arrival (DoA) estimation of a moving target after application of the conventional DFT (6) and the adapted DFT (8) with the DoA estimation of a static target. The simulated radar uses the parameters in Tab. I and a TDM MIMO array with 2 transmitters in a distance of $5 \lambda$ and 10 receivers with $\lambda / 2$-spacing. It forms a 20 elements uniform linear virtual array with element spacing $\lambda / 2$. All DoA estimations are done with a Bartlett beamformer [11].

The noise-free simulation considers a target in distance $30 \mathrm{~m}, \vartheta=15^{\circ}$, and the velocities $v=0$ and $v=18 \mathrm{~m} / \mathrm{s}$. Fig. 4 shows the DoA estimation on the range-Doppler cell that contains the target. The estimation for $v=0$ results in $15.2^{\circ}$, the closest bin to the actual DoA of $15^{\circ}$. This estimation is used as reference. When the range-Doppler spectrum is determined with (6) for $v \neq 0$, the motion-induced phase error corrupts the estimated DoA to $18.5^{\circ}$ and leads to a deformed spectrum. However, when the Doppler processing is done using (8), the estimation for $v=18 \mathrm{~m} / \mathrm{s}$ is identical to the static reference.

A measurement evaluation is done with a TDM MIMO radar with 2 transmitters and 10 receivers, and the modulation parameters in Tab. I. The virtual array is a uniform linear array with element spacing $0.545 \lambda$ that contains an overlapping element. The sensor is mounted on a car with a velocity of 


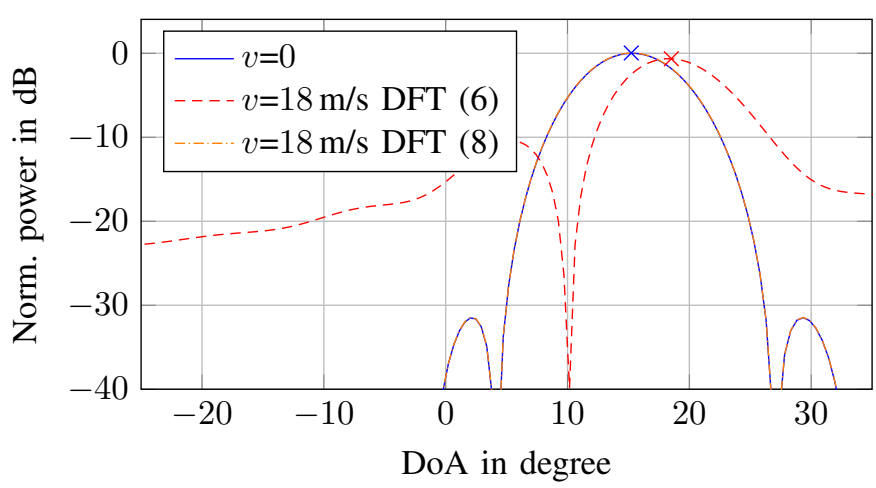

Fig. 4. Simulation of a single target at $15^{\circ}$ for the different velocities $v=0$ and $v=18 \mathrm{~m} / \mathrm{s}$. For $v=0$ no phase error occurs. In the case $v=18 \mathrm{~m} / \mathrm{s}$ and a Doppler processing with (6) the motion-induced phase error leads to a wrong DoA estimation and a deformed spectrum. In case of Doppler processing with the adjusted DFT (8), the DoA estimation is identical to that one without velocity.

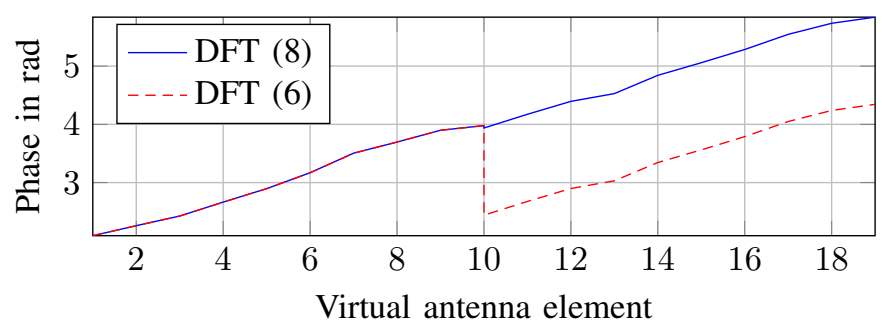

Fig. 5. Measured phases at the elements of the MIMO virtual array. The element at position 10 occurs twice in the virtual aperture.

approximately $18 \mathrm{~m} / \mathrm{s}$. The range-Doppler matrix is calculated with the conventional DFT processing (6) and the newly proposed processing (8). In the range-Doppler spectrum, a dominant target peak is selected for DoA estimation. Fig. 5 shows the phase of this peak at the virtual array positions. At position 10 (overlapping element), two phases are included in the plot. The virtual elements before this transition belong to the first transmitter, the other ones to the second transmitter. At the position of the overlapping element there is a phase discontinuity of $1.54 \mathrm{rad}$ for the conventional processing (6), due to the motion-induced phase error. The phase-corrected range-Doppler processing (8) does not show such a severe deviation. Instead, the phase values at the position of the overlapping elements are nearly identical.

Fig. 6 shows the corresponding DoA estimation for the following cases: range-Doppler processing with (6), rangeDoppler processing with (6) followed by phase correction with the overlapping element according to [6], DoA estimation using the data of a single transmitter (singe-input multipleoutput, SIMO), and range-Doppler processing with (8).

The application of (6) leads to two broad peaks in the DoA estimation. The overall maximum appears at the DoA of $1^{\circ}$. When the overlapping element is used for phase correction, the estimation results in one narrow peak at $3.9^{\circ}$ and the curve shape has significantly lower side lobes. The SIMO estimation has a smaller aperture and thus a worse resolution; however, the maximum of the DoA estimation of $4.3^{\circ}$ is very similar to the previous one. When the MIMO DoA estimation is done after range-Doppler processing with (8), the estimated DoA is

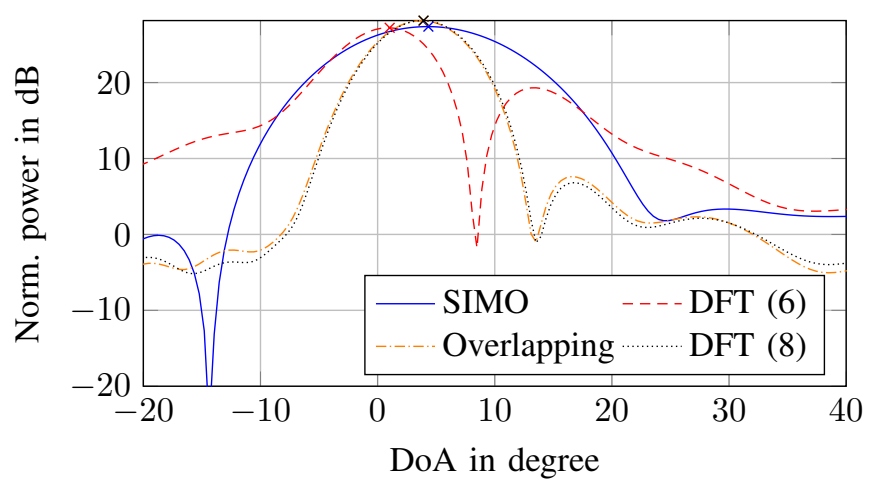

Fig. 6. DoA estimation for a measurement with velocity $18 \mathrm{~m} / \mathrm{s}$.

$3.9^{\circ}$ and the curve shape is nearly identical to the processing using the overlapping element; however, it does not require the overlapping element in the virtual aperture.

\section{CONCLUSION}

The paper introduced a range-Doppler processing to mitigate motion-induced phase errors in TDM MIMO radars without any additional hardware effort like an overlapping element. Simulations showed that the presented way of processing has the same DoA estimation performance in dynamic scenarios as a conventional processing in static scenarios. The compensation of the motion-induced phase error could also be verified with measurement results.

\section{ACKNOWLEDGMENT}

The authors want to thank the Daimler AG, Group Research and Advanced Engineering, Ulm, for their support during the measurements.

\section{REFERENCES}

[1] F. Belfiori et al., "TDMA X-band FMCW MIMO Radar for Short Range Surveillance Applications," in Proceedings of the 5th European Conference on Antennas and Propagation, Apr. 2011, pp. 483-487.

[2] S. Lutz and T. Walter, "Lens based 77 GHz TDM MIMO Radar Sensor for Angular Estimation in Multitarget Environments," in European Radar Conference, Oct. 2013, pp. 212-215.

[3] T. Spreng et al., "Wideband $120 \mathrm{GHz}$ to $140 \mathrm{GHz}$ MIMO Radar: System Design and Imaging Results," in European Microwave Conf., Sep. 2015.

[4] D. Bleh et al., "W-Band Time-Domain Multiplexing FMCW MIMO Radar for Far-Field 3-D Imaging," IEEE Trans. Microw. Theory Techn., no. 99, 2017.

[5] V. Winkler, "Range Doppler Detection for automotive FMCW Radars," in European Microwave Conference, Oct. 2007, pp. 1445-1448.

[6] C. Schmid et al., "Motion Compensation and Efficient Array Design for TDMA FMCW MIMO Radar Systems," in 6th European Conference on Antennas and Propagation (EUCAP), Mar. 2012, pp. 1746-1750.

[7] K. Rambach and B. Yang, "Colocated mimo radar: Cramer-rao bound and optimal time division multiplexing for doa estimation of moving targets," in Int. Conf. on Acoustics, Speech and Signal Processing, May 2013.

[8] D. Zoeke and A. Ziroff, "Phase Migration Effects in Moving Target Localization Using Switched MIMO Arrays," in European Radar Conference (EuRAD), Sep. 2015, pp. 85-88.

[9] J. Guetlein et al., "Switching Scheme for a FMCW-MIMO Radar on a Moving Platform," in European Radar Conference, Oct. 2012, pp. 91-94.

[10] K. Thurn et al., "Concept and implementation of a pll-controlled interlaced chirp sequence radar for optimized range-doppler measurements," IEEE Trans. Microw. Theory Techn., vol. 64, no. 10, Oct 2016.

[11] P. Häcker and B. Yang, "Single snapshot doa estimation," Advances in Radio Science, vol. 8, pp. 251-256, 2010. 